\title{
HARMONIC CLOSE-TO-CONVEX MAPPINGS
}

\author{
JAY M. JAHANGIRI ${ }^{1}$ \\ Kent State University \\ Department of Mathematics \\ Burton, OH 44021-9500 USA \\ E-mail: jay@geauga.kent.edu \\ HERB SILVERMAN \\ College of Charleston \\ Department of Mathematics \\ Charleston, SC 29424-0001 USA \\ E-mail: silvermanh@cofc.edu
}

(Received October, 1999; Revised May, 2000)

\begin{abstract}
Sufficient coefficient conditions for complex functions to be close-to-convex harmonic or convex harmonic are given. Construction of close-to-convex harmonic functions is also studied by looking at transforms of convex analytic functions. Finally, a convolution property for harmonic functions is discussed.
\end{abstract}

Key words: Harmonic, Convex, Close-to-Convex, Univalent.

AMS subject classifications: $30 \mathrm{C} 45,58 \mathrm{E} 20$.

\section{Introduction}

Harmonic functions are famous for their use in the study of minimal surfaces and also play important roles in a variety of problems in applied mathematics. Harmonic functions have been studied by differential geometers such as Choquet [2], Kneser [7], Lewy [8], and Rado [9]. Recent interest in harmonic complex functions has been triggered by geometric functions theorists Clunie and Sheil-Small [3].

A continuous function $f=u+i v$ is a complex-valued harmonic functions in a domain $\mathcal{D} \subset C$ if both $u$ and $v$ are real harmonic in $\mathcal{D}$. In any simply connected domain, we can write

$$
f=h+\bar{g},
$$

where $h$ and $g$ are analytic in $\mathcal{D}$. We call $h$ the analytic part and $g$ the co-analytic part of $f$. A necessary and sufficient conditions (see [3] or [8]) for $f$ to be locally univalent and sensepreserving in $\mathcal{D}$ is that $\left|h^{\prime}(z)\right|>\left|g^{\prime}(z)\right|$ in $\mathcal{D}$.

Denote by $\mathcal{S}_{\mathcal{H}}$ the class of functions $f$ of the form (1) that are harmonic univalent and sense-preserving in the unit disk $\Delta=\{z:|z|<1\}$ for which $f(0)=f_{z}(0)-1=0$. Thus we may write

${ }^{1}$ Dedicated to KSU Professor Richard S. Varga on his seventieth birthday. 


$$
h(z)=z+\sum_{n=2}^{\infty} a_{n} z^{n} \text { and } g(z)=\sum_{n=1}^{\infty} b_{n} z^{n} .
$$

Note that $\mathcal{S}_{\mathcal{H}}$ reduces to $\mathcal{S}$, the class of normalized univalent analytic functions if the coanalytic part of $f$ is zero. Since $h^{\prime}(0)=1>\left|g^{\prime}(0)\right|=\left|b_{1}\right|$ for $f \in \mathcal{S}_{\mathcal{H}}$, the function $\left(f-\overline{b_{1} f}\right) /\left(1-\left|b_{1}\right|^{2}\right)$ is also in $\mathcal{S}_{\mathcal{H}}$. Therefore, we may sometimes restrict ourselves to $\mathcal{S}_{\mathcal{H}}^{o}$, the subclass of $\mathcal{S}_{\mathcal{H}}$ for which $b_{1}=f_{\bar{z}}(0)=0$. In [3], it was shown that $\mathcal{S}_{\mathcal{H}}$ is normal and $\mathcal{S}_{\mathcal{H}}^{o}$ is compact with respect to the topology of locally uniform convergence. Some coefficient bounds for convex and starlike harmonic functions have recently been obtained by Avci and Zlotkiewicz [1], Jahangiri [5, 6], and Silverman [14].

In this paper, we give sufficient conditions for functions in $\mathcal{S}_{\mathcal{H}}$ to be close-to-convex harmonic or convex harmonic. We also construct close-to-convex harmonic functions by looking at transforms of convex analytic functions. Finally, we discuss a convolution property for harmonic functions.

In the sequel, unless otherwise stated, we will assume that $f$ is of the form (1) with $h$ and $g$ of the form (2).

\section{Convex and Close-to-Convex Mappings}

Let $\mathcal{K}, \mathcal{K}_{\mathcal{H}}$ and $\mathcal{K}_{\mathcal{H}}^{o}$ denote the respective subclasses of $\mathcal{S}, \mathcal{S}_{\mathcal{H}}$ and $\mathcal{S}_{\mathcal{H}}^{o}$ where the images of $f(\Delta)$ are convex. Similarly, $\mathcal{C}, \mathcal{C}_{\mathcal{H}}$ and $\mathcal{C}_{\mathcal{H}}^{o}$ denote the subclass of $\mathcal{S}, \mathcal{S}_{\mathcal{H}}$ and $\mathcal{S}_{\mathcal{H}}^{o}$ where the images of $f(\Delta)$ are close-to-convex. Recall that a domain $\mathcal{D}$ is convex if the linear segment joining any two points of $\mathcal{D}$ lies entirely in $\mathcal{D}$. A domain $\mathcal{D}$ is called close-to-convex if the complement of $\mathcal{D}$ can be written as a union of non-crossing half-lines. For other equivalent criteria, see [4].

Clunie and Sheil-Small[3] proved the following results.

Theorem A: If $h, g$ are analytic in $\Delta$ with $\left|h^{\prime}(0)\right|>\left|g^{\prime}(0)\right|$ and $h+\epsilon g$ is close-toconvex for each $\epsilon,|\epsilon|=1$, then $f=h+\bar{g}$ is harmonic close-to-convex.

Theorem B: If $f=h+\bar{g}$ is locally univalent in $\Delta$ and $h+\epsilon g$ is convex for some $\epsilon$, $|\epsilon| \leq 1$, then $f$ is univalent close-to-convex.

A domain $\mathcal{D}$ is called convex in the direction $\phi(0 \leq \phi<\pi)$ if every line parallel to the line through 0 and $e^{i \phi}$ has a connected intersection with $\mathcal{D}$. Such a domain is close-to-convex. The convex domains are those convex in every direction. We will also make use of the following result, which may be found in [3].

Theorem C: A function $f=h+\bar{g}$ is harmonic convex if and only if the analytic functions $h(z)-e^{i \phi} g(z), 0 \leq \phi<2 \pi$, are convex in the direction $\phi / 2$ and $f$ is suitably normalized.

The harmonic Koebe function $k_{0}=h+\bar{g} \in \mathcal{S}_{\mathcal{H}}^{o}$ is defined by $h(z)-g(z)=z /(1-z)^{2}$, $g^{\prime}(z)=z h^{\prime}(z)$, which leads to

$$
h(z)=\frac{z-\frac{1}{2} z^{2}+\frac{1}{6} z^{3}}{(1-z)^{3}}, g(z)=\frac{\frac{1}{2} z^{2}+\frac{1}{6} z^{3}}{(1-z)^{3}} .
$$

The function $k_{o}$ maps $\Delta$ onto the complex plane minus the real slit from $-1 / 6$ to $-\infty$. The coefficients of $k_{o}$ are $a_{n}=(2 n+1)(n+1) / 6$ and $b_{n}=(2 n-1)(n-1) / 6$. These coefficient bounds are known to be extremal for the subclass of $\mathcal{S}_{\mathcal{H}}^{o}$ consisting of typically real functions (e.g., see [3]) and functions that are either starlike or convex in one direction (e.g., see [12]). It is not known if the coefficients of $k_{o}$ are extremal for all of $\mathcal{S}_{\mathcal{H}}^{o}$.

Necessary coefficient conditions were found in [3] for functions to be in $\mathcal{C}_{\mathcal{H}}$ and $\mathcal{K}_{\mathcal{H}}$. We now give some sufficient condition for functions to be in these classes. But first we need the following results. See, for example, [13]. 
Lemma 1: If $q(z)=z+\sum_{n=2}^{\infty} c_{n} z^{n}$ is analytic in $\Delta$, then $q$ maps onto a starlike domain if $\sum_{n=2}^{\infty} n\left|c_{n}\right| \leq 1$ and onto a convex domains if $\sum_{n=2}^{\infty} n^{2}\left|c_{n}\right| \leq 1$.

\section{Main Results}

Theorem 1: If $f=h+\bar{g}$ with

$$
\sum_{n=2}^{\infty} n\left|a_{n}\right|+\sum_{n=1}^{\infty} n\left|b_{n}\right| \leq 1,
$$

then $f \in \mathcal{C}_{\mathcal{H}}$. The result is sharp.

Proof: In view of Theorem A, we need only prove that $h+\epsilon g,|\epsilon|=1$, is close-toconvex. It suffices to show that

$$
t(z)=\frac{h+\epsilon g}{1+\epsilon b_{1}}=z+\sum_{n=2}^{\infty}\left(\frac{a_{n}+\epsilon b_{n}}{1+\epsilon b_{1}}\right) z^{n} \in \mathcal{C}
$$

Since

$$
\sum_{n=2}^{\infty} n\left|\frac{a_{n}+\epsilon b_{n}}{1+\epsilon b_{1}}\right| \leq \sum_{n=2}^{\infty} \frac{n\left(\left|a_{n}\right|+\left|b_{n}\right|\right)}{1-\left|b_{1}\right|} \leq 1
$$

if and only if (3) holds, $t(z)$ maps $\Delta$ onto a starlike domain and consequently $t(z) \in \mathcal{C}$.

To see that the upper bound in (3) cannot be extended to $1+\delta, \delta>0$, we note that the function $f(z)=z+\frac{1+\delta}{n} z^{n}$ is not univalent in $\Delta$.

Theorem 2: If $f$ is locally univalent with $\sum_{n=2}^{\infty} n^{2}\left|a_{n}\right| \leq 1$, then $f \in \mathcal{C}_{\mathcal{H}}$.

Proof: Take $\epsilon=0$ in Theorem B and apply Lemma 1 .

Corollary: If $\sum_{n=2}^{\infty} n^{2}\left|a_{n}\right| \leq 1$ and $\left|g^{\prime}(z)\right| \leq 1 / 2, z \in \Delta$, then $f \in \mathcal{C}_{\mathcal{H}}$.

Proof: The function $f$ is locally univalent if $\left|h^{\prime}(z)\right|>\left|g^{\prime}(z)\right|$ for $z \in \Delta$. Since $2 \sum_{n=2}^{\infty} n\left|a_{n}\right| \leq \sum_{n=2}^{\infty} n^{2}\left|a_{n}\right| \leq 1$, we have $h^{\prime}(z)>1-\sum_{n=2}^{\infty} n\left|a_{n}\right| \geq 1 / 2$.

We next give a sufficient coefficient condition for $f$ to be convex harmonic.

Theorem 3: If

$$
\sum_{n=2}^{\infty} n^{2}\left|a_{n}\right|+\sum_{n=1}^{\infty} n^{2}\left|b_{n}\right| \leq 1
$$

then $f \in \mathcal{K}_{\mathcal{H}}$. The result is sharp.

Proof: By Theorem C, it suffices to show that $h-e^{i \phi} g$ is convex in $\Delta$. Set

$$
s(z)=\frac{h-e^{i \phi} g}{1-e^{i \phi} b_{1}}=z+\sum_{n=2}^{\infty}\left(\frac{a_{n}-e^{i \phi} b_{n}}{1-e^{i \phi} b_{1}}\right) z^{n} .
$$

Since

$$
\sum_{n=2}^{\infty} n^{2}\left|\frac{a_{n}-e^{i \phi} b_{n}}{1-e^{i \phi} b_{1}}\right| \leq \sum_{n=2}^{\infty} \frac{n^{2}\left(\left|a_{n}\right|+\left|b_{n}\right|\right)}{1-\left|b_{1}\right|} \leq 1
$$

if and only if (4) holds, we see from Lemma 1 that $s(z) \in \mathcal{K}$ and consequently $f \in \mathcal{K}_{\mathcal{H}}$.

The function $f(z)=z+\frac{1+\delta}{n^{2}} z^{n}, \delta>0$, shows that the upper bound in (4) cannot be improved. 
Remark: The coefficient bound given in Theorem 3 can also be found in [5] and [14]. However, our approach in this paper is different from those given in [5] and [14].

Remark: The well-known results for univalent functions that $f$ is convex if and only if $z f^{\prime}$ is starlike does not carry over to harmonic univalent functions. See [12]. Hence, we cannot conclude from Theorem 3 that (3) is a sufficient condition for $f$ to map $\Delta$ onto a starlike domain. Nevertheless, we believe this to be the case. See [5, 6, 14].

We now introduce a class of harmonic close-to-convex functions that are constructed from convex analytic functions.

Theorem 4: If $h(z) \in \mathcal{K}$ and $w(z)$ is a Schwartzfunction, then

$$
f(z)=h(z)+\overline{\int_{0}^{z} w(t) h^{\prime}(t) d t} \in \mathcal{C}_{\mathcal{H}}^{o}
$$

Proof: Write $g^{\prime}(z)=w(z) h^{\prime}(z)$. Now for each $\epsilon,|\epsilon|=1$, we observe that

$$
\operatorname{Re} \frac{h^{\prime}(z)=\epsilon g^{\prime}(z)}{h^{\prime}(z)}=\operatorname{Re}(1+\epsilon w(z)) \geq 1-|z|>0, z \in \Delta .
$$

Consequently, $h+\epsilon g$ is close-to-convex and the result follows from Theorem A.

Remark: If we only require that $w$ in Theorem 4 be analytic with $|w(z)|<1, z \in \Delta$, then we may conclude that $f \in \mathcal{C}_{\mathcal{H}}$.

Corollary: If $h \in \mathcal{K}$ and $n$ is a positive integer, then

$$
f_{n}(z)=\int_{0}^{z}\left(\frac{h(t)}{t}\right)^{2} d t+\overline{\int_{0}^{z} t^{n-2} h^{2}(t) d t} \in \mathcal{C}_{\mathcal{H}}^{o}
$$

Proof: A result of Sheil-Small [10] shows that $\int_{0}^{z}(h(t) / t)^{2} d t \in \mathcal{K}$ whenever $h \in \mathcal{K}$. Set $w(z)=z^{n}$ in Theorem 4 , and the result follows.

We now give some examples from Theorem 4.

Example 1: Suffridge [15] showed for the partial sums $p_{n}(z)$ of $e^{1+z}=\sum_{k=0}^{\infty}(1+z)^{k} / k$ ! that

$$
C_{n}(z)=\frac{p_{n}(z)-p_{n}(0)}{p_{n}^{\prime}(0)}=\sum_{k=1}^{n}\left(\frac{\sum_{l=0}^{n-k} \frac{1}{1 !}}{\sum_{l=0}^{n-1} \frac{1}{l !}}\right) \frac{1}{k !} z^{k} \in \mathcal{K} .
$$

Setting $w(z)=z$ in Theorem 4, we see that

$$
f(z)=\sum_{k=1}^{n}\left(\frac{\sum_{l=0}^{n-k} \frac{1}{l !}}{\sum_{l=0}^{n-1} \frac{1}{l 1}}\right)\left(\frac{(k+1) z^{k}+k z^{k+1}}{(k+1) !}\right) \in \mathcal{C}_{\mathcal{H}}^{o} .
$$

Example 2: Since $h_{k}(z)=z+z^{k} / k^{2} \in \mathcal{K}$, we get from the Corollary that

$$
f_{k, n}(z)=z+\frac{2}{k^{3}} z^{k}+\frac{1}{k^{4}(2 k-1)} z^{2 k-1}+\overline{\frac{z^{n+1}}{n+1}+\frac{2 z^{n+k}}{k^{2}(n+k)}+\frac{z^{2 k+n-1}}{k^{4}(2 k+n-1)}} \in \mathcal{C}_{\mathcal{H}}^{o}
$$

for $k=2,3, \ldots$, and $n=1,2, \ldots$

Example 3: Set $h(z)=z /(1-z)$ and $w(z)=z$ in Theorem 4. Then

$$
f(z)=\frac{z}{1-z}+\overline{\int_{0}^{z} \frac{t}{(1-t)^{2}} d t}=2 \operatorname{Re} \frac{z}{1-z}+\log (1-\bar{z}) \in \mathcal{C}_{\mathcal{H}}^{o} .
$$

We can actually state a more general result for which Example 3 is a special case. 
Theorem 5: If $b(z)$ is analytic with $|b(z)|<1 /|1-z|^{2}, z \in \Delta$, then

$$
f(z)=\frac{z}{1-z}+\overline{\int_{0}^{z} b(t) d t} \in \mathcal{C}_{\mathcal{H}}
$$

Proof: Set $h(z)=z /(1-z)$ and $g(z)=\int_{o}^{z} b(t) d t$. Then $\left|h^{\prime}(z)\right|=(1 / \mid 1-$ $\left.\left.z\right|^{2}\right)>\left|g^{\prime}(z)\right|=|b(z)|$, so that $f$ is locally univalent. Set $\epsilon=0$ in Theorem B, and the result follows.

Corollary: If $b(z)$ is analytic with $|b(z)| \leq 1 / 4, z \in \Delta$, then

$$
\frac{z}{1-z}+\overline{\int_{0}^{z} b(t) d t} \in \mathcal{C}_{\mathcal{H}}
$$

\section{Convolution Condition}

The convolution of two harmonic functions $f_{1}(z)=z+\sum_{n=2}^{\infty} a_{n} z^{n}+\sum_{n=1}^{\infty} b_{n} \bar{z}^{n}$ and $f_{2}(z)=z+\sum_{n=2}^{\infty} A_{n} z^{n}+\sum_{n=1}^{\infty} B_{n} \bar{z}^{n}$ is defined by

$$
f_{1}(z) * f_{2}(z)=\left(f_{1} * f_{2}\right)(z)=z+\sum_{n=2}^{\infty} a_{n} A_{n} z^{n}+\sum_{n=1}^{\infty} b_{n} B_{n} \bar{z}^{n} .
$$

In [3], it was shown for $\phi \in \mathcal{K}$ and $f \in \mathcal{K}_{\mathcal{H}}$ that $(\phi+\epsilon \bar{\phi}) * f \in \mathcal{C}_{\mathcal{H}}(|\epsilon| \leq 1)$. We given an example to show that $\mathcal{K}$ cannot be replaced by $S^{*}(\alpha), 0 \leq \alpha<1$, the family of functions starlike of order $\alpha$.

Set

$$
\phi(z)=z+\frac{1-\alpha}{n-\alpha} z^{n} \in S^{*}(\alpha), h(z)=\frac{z-z^{2} / 2}{(1-z)^{2}}, g(z)=\frac{-z^{2} / 2}{(1-z)^{2}} .
$$

Then $f=h+\bar{g} \in \mathcal{K}_{\mathcal{H}}$, see [3]. Setting $\epsilon=0$ in $(\phi+\epsilon \bar{\phi}) * f$ we obtain

$$
\begin{gathered}
\phi * f=\phi *(h+\bar{g})=\phi * h=\left(z+\frac{1-\alpha}{n-\alpha} z^{n}\right) *\left(z+\sum_{n=2}^{\infty} \frac{n+1}{2} z^{n}\right) \\
=z+\frac{(1-\alpha)(n+1)}{2(n-\alpha)} z^{n}
\end{gathered}
$$

which is not even univalent for $n>2 \alpha /(1-\alpha)$.

\section{References}

[1] Avci, Y. and Zlotkiewicz, E., On harmonic univalent mappings, Ann. Univ. Marie Curie-Sklodowska Sect. A 44 (1991), 1-7.

[2] Choquet, G., Sur un type de transformation analytique généralisant la représentation conforme et définie au moyen de fonctions harmoniques, Bull. Sci. Math. 69:2 (1945), 156-165.

[3] Clunie, J. and Sheil-Small, T., Harmonic univalent functions, Ann. Acad. Sci. Fenn. Ser. A.I Math 9 (1984), 3-25.

[4] Duren, P.L., Univalent Functions, Springer-Verlag, New York 1983. 
[5] Jahangiri, J.M., Coefficient bounds and univalence criteria for harmonic functions with negative coefficients, Ann. Univ. Marie Curie-Sklodowska Sect. A 52 (1998), 57-66.

[6] Jahangiri, J.M., Harmonic functions starlike in the unit disk, J. Math. Anal. Appl. 235 (1999), 470-477.

[7] Kneser, H., Lösung der aufgabe 41, Jahresber, Deutsch. Math.-Verein. 35 (1926), 123124.

[8] Lewy, H., On the non-vanishing of the Jacobian in certain one-to-one mappings, Bull. Amer. Math. Soc. 42 (1936), 689-692.

[9] Rado, T., Aufgabe 41, Jahresber. Deutsch. Math.-Verein. 35 (1926), 49.

[10] Sheil-Small, T., On convex univalent functions, J. London Math. Soc. 2:1 (1969), 483492.

[11] Sheil-Small, T., On linear accessibility and the conformal mapping of convex domains, J. Analyse Math. 25 (1972), 259-276.

[12] Sheil-Small, T., Constant for planar harmonic mappings, J. London Math. Soc. 2:42 (1990), 237-248.

[13] Silverman, H., Univalent functions with negative coefficients, Proc. Amer. Math. Soc. 51 (1975), 109-116.

[14] Silverman, H., Harmonic univalent functions with negative coefficients, J. Math. Anal. Appl. 220 (1998), 283-289.

[15] Suffridge, T.J., On a family of convex polynomials, Rocky Mountain J. Math. 22:1 (1992), 387-391. 


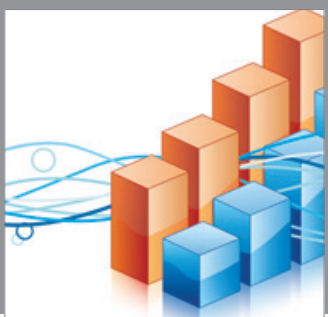

Advances in

Operations Research

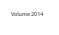

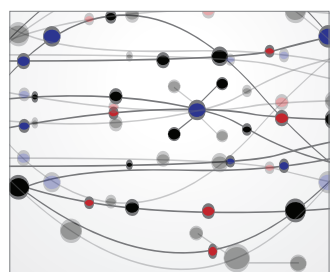

\section{The Scientific} World Journal
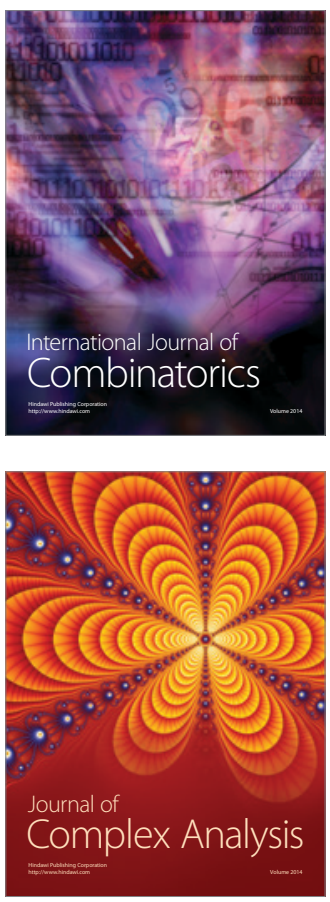

International Journal of

Mathematics and

Mathematical

Sciences
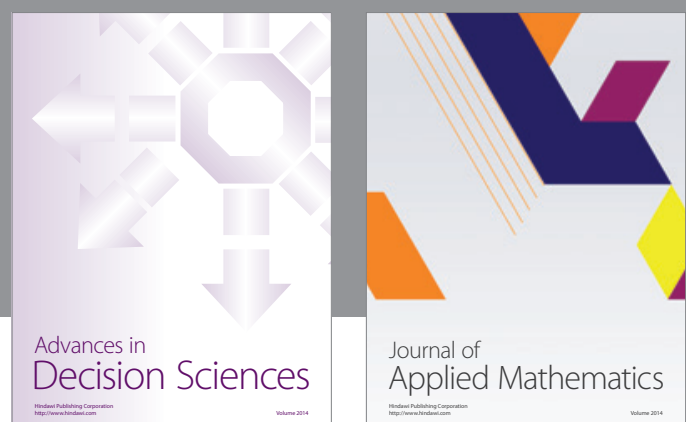

Journal of

Applied Mathematics
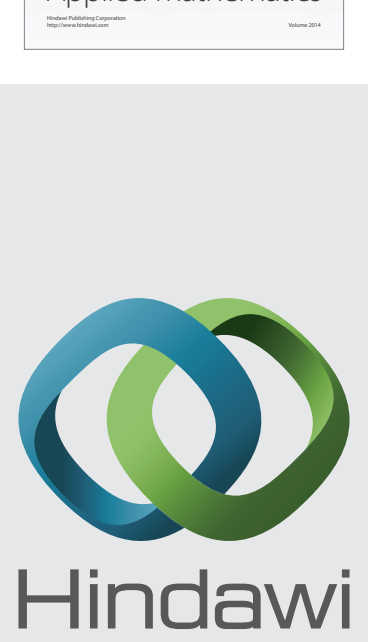

Submit your manuscripts at http://www.hindawi.com
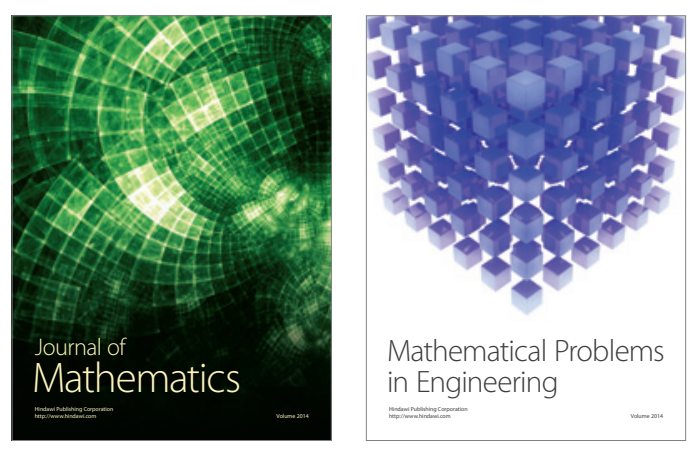

Mathematical Problems in Engineering
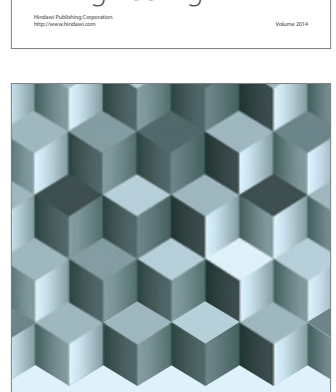

Journal of

Function Spaces
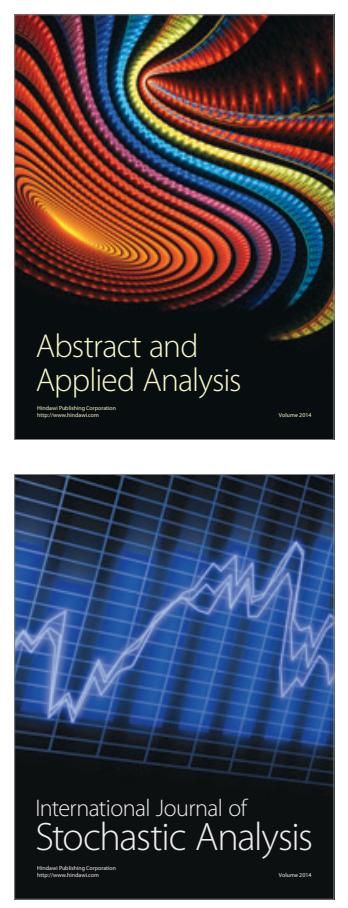

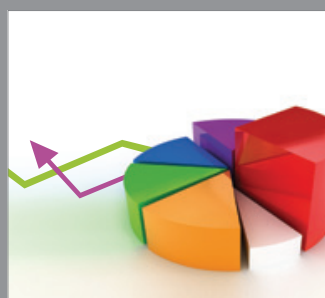

ournal of

Probability and Statistics

Promensencen
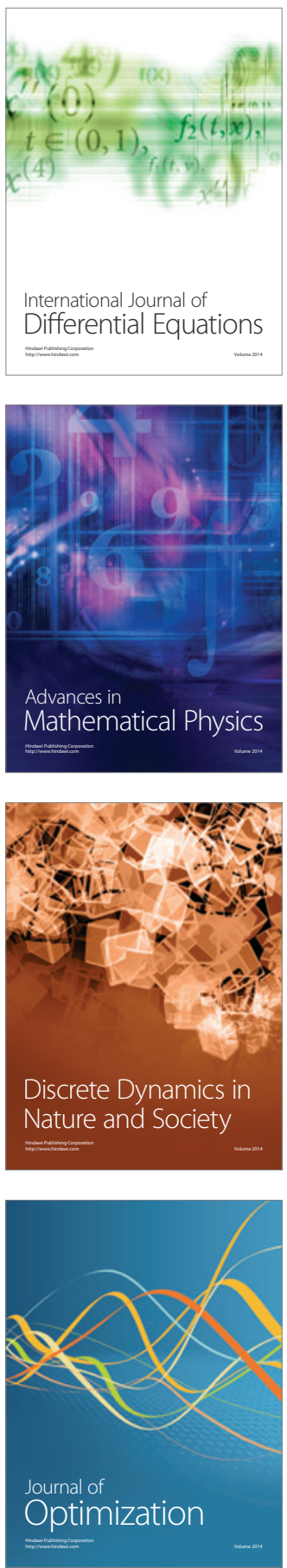